\title{
Prognostic value of serum apolipoprotein A-I in locally advanced cervical squamous cell carcinoma treated with radical chemoradiotherapy
}

\author{
Hui Chang $^{1,2 \#}$, Kai Chen ${ }^{1,2 \#}$, Yan-Zhu Lin ${ }^{1,2}$, Ya-Lan Tao ${ }^{1,2}$, Yang Liu ${ }^{1,2}$, Xin-Ping Cao ${ }^{1,2}$, Wei-Jun Ye,2 \\ ${ }^{1}$ Department of Radiation Oncology, Sun Yat-sen University Cancer Center, Guangzhou 510060, China; ${ }^{2}$ State Key Laboratory of Oncology in \\ South China, Collaborative Innovation Center for Cancer Medicine, Guangzhou 510060, China \\ Contributions: (I) Conception and design: H Chang, K Chen, XP Cao, WJ Ye; (II) Administrative support: XP Cao, WJ Ye; (III) Provision of study \\ materials or patients: H Chang, K Chen, YZ Lin, YL Tao, Y Liu; (IV) Collection and assembly of data: H Chang, K Chen; (V) Data analysis and \\ interpretation: H Chang, K Chen; (VI) Manuscript writing: All authors; (VII) Final approval of manuscript: All authors. \\ \#These authors contributed equally to this work. \\ Correspondence to: Prof. Xin-Ping Cao; Prof. Wei-Jun Ye. Department of Radiation Oncology, Sun Yat-sen University Cancer Center, 651 Dongfeng \\ Road East, Guangzhou 510060, China. Email: caoxp@sysucc.org.cn; yewj1968@126.com.
}

\begin{abstract}
Background: Distant metastasis (DM) remains the major cause of death from locally advanced cervical squamous cell carcinoma (CSCC). This study aimed to evaluate the predictive value of pretreatment serum lipids on DM and death, in patients with locally advanced CSCC.

Methods: In this study, a total of 331 consecutive patients with stages IIB-IVA CSCC and completed record of radical concurrent chemoradiotherapy (CCRT) were involved between January 2011 and June 2013. Pretreatment serum lipid indexes including total cholesterol (TC), triglyceride (TG), high-density lipoprotein cholesterol (HDL-c), low-density lipoprotein cholesterol (LDL-c), apolipoprotein A-I (apoAI) and apolipoprotein B (apoB) were analyzed for their association with the overall survival (OS), the localrecurrence-free survival (RFS), the distant-metastasis-free survival (MFS) and the disease-free survival (DFS), together with clinical features [age, stage, parametrial invasion, tumor diameter, lymph node metastasis, hemoglobin, squamous cell carcinoma (SCC) antigen and carbohydrate antigen 125 (CA125)].

Results: After univariate and multivariate analysis, serum apoAI level $(\geq 1.2$ vs. $<1.2 \mathrm{~g} / \mathrm{L})$ emerged as an independent predictor of the OS [hazard ratio $(\mathrm{HR})=0.500,95 \%$ confidence interval $(\mathrm{CI}), 0.321-0.778$ ], the MFS (HR =0.503, 95\% CI, 0.323-0.784) and DFS (HR =0.521 95\% CI, 0.338-0.803).

Conclusions: For locally advanced CSCC treated with CCRT, low pretreatment level of serum apoAI could predict high risk of DM and poor prognosis. This finding might help clinicians to direct therapy for eradicating DM and conduct clinical trials on apoAI-targeting anti-tumor therapies.
\end{abstract}

Keywords: Cervical cancer; apolipoprotein A-I (apoAI); distant metastasis (DM); survival

Submitted Jun 29, 2018. Accepted for publication Nov 05, 2018.

doi: $10.21037 /$ tcr.2018.11.10

View this article at: http://dx.doi.org/10.21037/tcr.2018.11.10

\section{Introduction}

The cervical squamous cell carcinoma (CSCC) accounts for nearly two-thirds of all cervical cancer cases, which is a common malignancy threatening the health of Chinese females $(1,2)$. Currently, patients with early (stages I-IIA) CSCC are mainly treated with radical surgery and could achieve a 5-year overall survival (OS) of $94.6 \%$ (3). Oppositely, even after multimodality treatment based on radiotherapy, the 5 -year OS of patients with locally advanced (stages IIB-IVA) CSCC still emerges as $65.8-75.7 \%$ (4). Distant metastasis (DM), rather than local recurrence (LR), is the main cause of treatment failure in these patients. 
Totally $19.2-22.5 \%$ of them will develop DM $(4,5)$. In other words, prognosis of locally advanced CSCC could be improved through eradicating DM.

Intensified systemic treatments, such as adjuvant chemotherapy (ACT) after concurrent chemoradiotherapy (CCRT), were tried to reduce DM from cervical cancer, including CSCC. But insufficient evidence is found to support routine use of ACT, which is convinced to bring more haematological toxicities (6). So it is necessary to identify the cases really at high risk of DM and in need of ACT. The golden standard to predict prognosis of cervical cancer is now the International Federation of Gynecology and Obstetrics (FIGO) stage (7). However, controversies are often seen between the clinical outcomes of patients and their FIGO stage (8). The possible explanation may be that the FIGO staging system is based merely on anatomical range of tumor invasion. Functional factors which reflect biological behavior of tumor cells are not taken into consideration.

Serum lipids are practical functional biomarkers used in daily clinical work. Recent studies proved that pretreatment level of some serum lipoproteins and their core component, apolipoproteins, were associated with the prognosis of many solid tumors (9-12). However, there are few researches reporting how these factors influence DM and death from CSCC. Hence, this study aimed to evaluate the prognostic value of serum lipid profile in patients with locally advanced CSCC.

\section{Methods}

\section{Patient selection}

Patients with pathologically diagnosed and previously untreated CSCC in our hospital from January 1st 2011 to June 30th 2013 were initially considered. The patients were enrolled into this study if they had age $\geq 18$ and $<75$ years old, stages IIB-IVA disease at diagnosis [FIGO criteria ver. 2009 (7)], and complete record of radical CCRT. The exclusion criteria included: (I) Karnofsky performance score $<70$; (II) severe hematopoietic, heart, lung, liver or kidney dysfunction unsuitable for radiotherapy; (III) prior history of other malignancies; (IV) hyperlipidemia, diabetes and regular intake of lipid-modulating drugs, such as niacin and statins.

All patients had detailed medical records, including but not limited to pretreatment complete blood count, serum lipid assessment, serum level of squamous cell carcinoma (SCC) antigen and carbohydrate antigen 125 (CA125), magnetic resonance imaging (MRI) of abdomen and pelvis, computed tomography (CT) of neck and chest, and wholebody bone scan or positron emission tomography (PET).

\section{Treatment strategies}

All the patients were treated according to the clinical practice guidelines of our hospital and the National Comprehensive Cancer Network. The procedure of radiotherapy consisted of an external beam radiation (EBRT) and a high-dose rate brachytherapy (BT). The ERBT was performed with a 3-dimensional conformal radiotherapy (3DCRT) or intensity-modulated radiotherapy (IMRT) technique. The gross tumor volume (GTV) was the enlarged lymph nodes visible on MRI. The clinical target volume (CTV) covered the GTV with a radial margin of $2 \mathrm{~cm}$, and included high-risk regions of lymphatic drainage (the paracervical, the obturator, the presacral, and the lateral, internal and common iliac lymph node regions). The EBRT was done in a conventional fractionation (2 Gy per fraction, 1 fraction per day, 5 days per week), in which the total dose for GTV and CTV were 60 and 45 Gy respectively. The regimen of chemotherapy concurrently with ERBT was single-agent cisplatin of $40 \mathrm{mg} / \mathrm{m}^{2}$ weekly (totally 4-5 weeks). After EBRT, 2-dimensional BT (iridium 192, microSelectron, Nucletron) was performed one fraction per week for 6 weeks. In each fraction, a dose of 6 Gy was administered to point A.

\section{Serum lipid profile}

Serum lipid profile of each patient was tested one week before treatment, including level of total cholesterol (TC), triglyceride (TG), high-density lipoprotein cholesterol (HDL-c), low-density lipoprotein cholesterol (LDL-c), apolipoprotein A-I (apoAI) and apolipoprotein B (apoB). The testing was performed by using a LABOSPECT 008 automatic biochemistry analyzer and its matching detection kit (HITACHI, Tokyo, Japan), according to the manufacturer's protocols. The abnormality of each index was determined on basis of our hospital's cutoff value standard. The upper limit of TC, TG LDL-c and apoB were 6.47, 1.7, $3.1 \mathrm{mmol} / \mathrm{L}$ and $1.1 \mathrm{~g} / \mathrm{L}$, respectively. And the lower limit of HDL-c and apoAI were $1.29 \mathrm{mmol} / \mathrm{L}$ and $1.2 \mathrm{~g} / \mathrm{L}$, respectively.

\section{Follow-up}

After treatment, the patients were followed up every 3 months from the 1 st to the 3 rd years, through outpatient 
Table 1 Baseline clinical profiles of the eligible patients

\begin{tabular}{|c|c|}
\hline Characteristic & Value \\
\hline Age at diagnosis (years) & 55 [27-75] \\
\hline \multicolumn{2}{|l|}{ No. of patients by stage (\%) } \\
\hline IIB & $188(56.8)$ \\
\hline IIIA & $38(11.5)$ \\
\hline IIIB & $99(29.9)$ \\
\hline IVA & $6(1.8)$ \\
\hline Tumor diameter $(\mathrm{cm})$ & $4.8(1.8-8.7)$ \\
\hline \multicolumn{2}{|l|}{$\begin{array}{l}\text { No. of patients by parametrial } \\
\text { invasion (\%) }\end{array}$} \\
\hline Yes & $263(79.5)$ \\
\hline No & $68(20.5)$ \\
\hline \multicolumn{2}{|l|}{$\begin{array}{l}\text { No. of patients by lymph node } \\
\text { metastasis (\%) }\end{array}$} \\
\hline Yes & $197(59.5)$ \\
\hline No & $134(40.5)$ \\
\hline Hemoglobin (g/L) & $120.50(53.00-158.00)$ \\
\hline Total cholesterol (mmol/L) & $5.02(1.29-30.60)$ \\
\hline Triglyceride (mmol/L) & $1.24(0.44-7.24)$ \\
\hline $\begin{array}{l}\text { High-density lipoprotein cholesterol } \\
(\mathrm{mmol} / \mathrm{L})\end{array}$ & $1.21(0.15-4.50)$ \\
\hline $\begin{array}{l}\text { Low-density lipoprotein cholesterol } \\
(\mathrm{mmol} / \mathrm{L})\end{array}$ & $3.03(0.93-9.77)$ \\
\hline Apolipoprotein A-I (g/L) & $1.22(0.25-1.79)$ \\
\hline Apolipoprotein B (g/L) & $0.95(0.38-2.18)$ \\
\hline $\begin{array}{l}\text { Squamous cell carcinoma antigen } \\
(\mathrm{mg} / \mathrm{L})\end{array}$ & $5.10(0.00-70.00)$ \\
\hline Carbohydrate antigen $125(\mathrm{U} / \mathrm{mL})$ & $15.74(0.00-7473.00)$ \\
\hline
\end{tabular}

Continuous and categorical data were presented as median with range and as number with percentage (\%), respectively.

interview. The main contents of outpatient interview included complete physical examination, cervicothoracic CT, abdominopelvic MRI, serum SCC antigen and CA125 assessment, and whole-body bone scan (or PET). From the 4th to the 5 th years, follow-up was made semi-annually through outpatient interview or telephone. After the 5th year, follow-up was made annually until death or March 31st 2018, whichever came first. Causes of death were confirmed by death certificates, which were supplemented with medical records if necessary.

\section{Statistical analysis}

The primary endpoint of this study was the OS, which referred to the percentage of the patients who were alive after a certain time period from pathological diagnosis. And the secondary endpoints included the local-recurrence-free survival (RFS), the distant-metastasis-free survival (MFS) and the disease-free survival (DFS). These 3 endpoints were defined as the percentage of patients who had no corresponding events after a certain time period from diagnosis. The events for the RFS and the MFS were LR and DM, respectively. And the events for the DFS included death, LR and DM.

The candidate factors for predicting the survivals included age, tumor diameter $(\leq 4 v s .>4 \mathrm{~cm})$, lymph node metastasis (no $v s$. yes), parametrial involvement (no vs. yes), hemoglobin ( $\geq 120$ vs. $<120 \mathrm{~g} / \mathrm{L})$, SCC antigen $(\leq 1.5$ vs. $>1.5 \mathrm{mg} / \mathrm{L})$, CA125 $(\leq 35$ vs. $>35 \mathrm{U} / \mathrm{mL})$, TC ( $\leq 6.47 v s .>6.47 \mathrm{mmol} / \mathrm{L}), \mathrm{TG}(\leq 1.7 v s .>1.7 \mathrm{mmol} / \mathrm{L})$, LDL-c $(\leq 3.1 v s .>3.1 \mathrm{mmol} / \mathrm{L})$, HDL-c $(\geq 1.29$ $v s .<1.29 \mathrm{mmol} / \mathrm{L})$, apoAI $(\geq 1.2 v s .<1.2 \mathrm{~g} / \mathrm{L})$ and apoB $(\leq 1.1 v s .>1.1 \mathrm{~g} / \mathrm{L})$. The cutoff value of the age was the median age of the whole cohort.

The factors above were firstly tested for their impact on the OS, the RFS, the MFS and the DFS, through a univariate survival analysis based on Kaplan-Meier approach and logrank test. The factors exhibiting statistical significance were then tested their predictive independence, through a multivariate analysis based on Cox proportional hazards model. The hazard ratio (HR) and 95\% confidence interval (CI) of each independent prognosticator were calculated.

Finally, serum lipid indexes exhibiting predictive independence were analyzed for their correlation with other clinical characteristics, based on Spearman's correlation analysis.

The statistical analyses were all made using IBM SPSS Statistics 23.0 (IBM Co., Armonk, New York, USA). A difference with a two-sided $\mathrm{P}$ value of less than 0.05 was considered to be statistically significant.

\section{Results}

\section{Baseline characteristics}

A total of 331 eligible patients with locally advanced CSCC were enrolled consecutively between January 1st 2011 and June 30th 2013. Of those, there were 188 (56.8\%), $38(11.5 \%), 99(29.9 \%)$ and $6(1.8 \%)$ cases with stages IIB, IIIA, IIIB and IVA disease, respectively. The baseline clinical characteristics of the patients were shown in Table 1. The median age of these patients was 
A

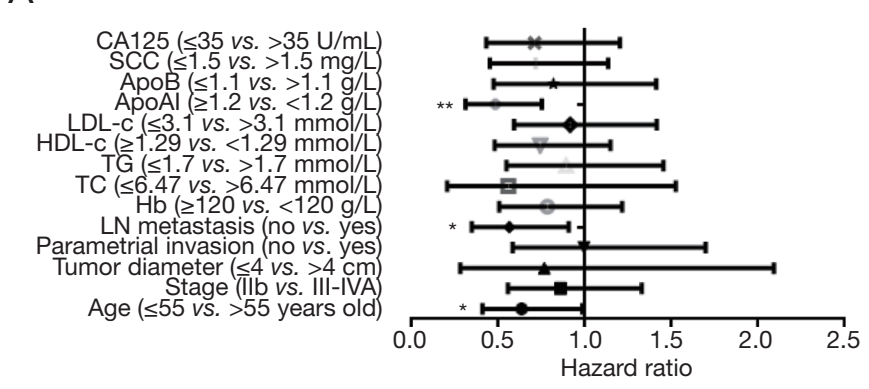

C

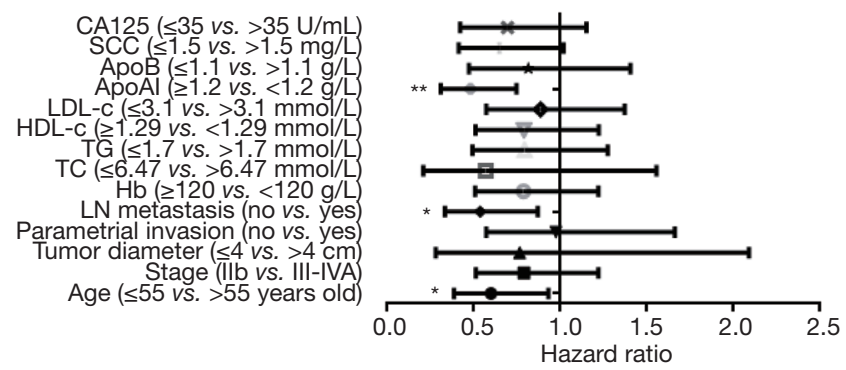

B Local-recurrence-free survival

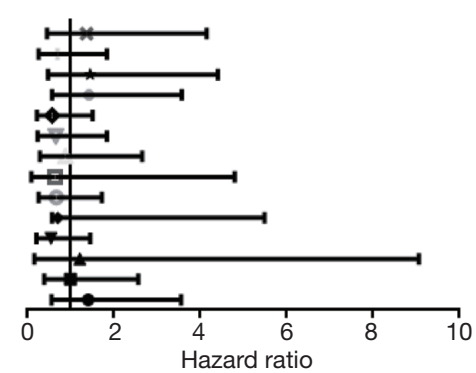

D

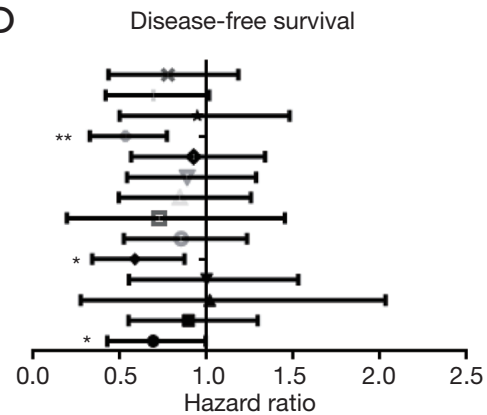

Figure 1 The results of univariate survival analysis. (A) overall survival; (B) local-recurrence-free survival; (C) distant-metastasis-free survival; (D) disease-free survival. * $\mathrm{P}<0.05$; ** $\mathrm{P}<0.01$. LN, lymph node; Hb, hemoglobin; TC, total cholesterol; TG, triglyceride; HDL-c, high-density lipoprotein cholesterol; LDL-c, low-density lipoprotein cholesterol; apoAI, apolipoprotein A-I; apoB, apolipoprotein B; SCC, squamous cell carcinoma antigen; CA125, carbohydrate antigen 125.

55 (range, 27-75) years old. So the cutoff value of the age was $55(\leq 55 v s .>55)$ years old.

\section{Survival analysis}

Until March 31st 2018, the median follow-up time of the patients was 49 (range, 7-85) months. Totally 52 of 331 patients $(15.7 \%)$ were lost to follow up. LR and $\mathrm{DM}$ were seen in $18(5.4 \%)$ and $83(25.1 \%)$ patients, respectively. The 3 most common sites of DM were lung $(22.7 \%)$, bone $(21.5 \%)$ and liver $(10.6 \%)$. There were 81 cancer-related deaths (24.5\%), including 67 cases $(20.2 \%)$ died from DM, and 14 cases (4.2\%) died from LR + DM.

The results of univariate analysis were shown in Figure 1. Age $\leq 55$ years old, no lymph node metastasis and apoAI $\geq 1.2 \mathrm{~g} / \mathrm{L}$ appeared as factors associated with a better OS (P values were $0.040,0.018$ and 0.001 , respectively; Figure 1A), MFS (P values were 0.023, 0.012 and 0.001 , respectively; Figure $1 C$ ) and DFS (P values were 0.042, 0.012 and 0.002 , respectively; Figure $1 D)$. SCC $\leq 1.5 \mathrm{mg} / \mathrm{L}$ was showed to bring a marginally better MFS $(\mathrm{P}=0.060)$ and DFS $(\mathrm{P}=0.057)$, though statistical significance was not achieved. Other serum lipid indexes, including TC, TG, LDL-c, HDL-c and apoB, failed to exhibit predictive ability on the OS, the RFS, the MFS or the DFS.

Through multivariate analysis (Table 2), age, lymph node metastasis and apoAI maintained as independent predictors of the OS (P values were $0.016,0.012$ and 0.002 , respectively), the MFS (P values were $0.008,0.007$ and 0.002 , respectively) and the DFS ( $\mathrm{P}$ values were $0.019,0.008$ and 0.003 , respectively). The HRs of apoAI $(\geq 1.2$ vs. $<1.2 \mathrm{~g} / \mathrm{L})$ for predicting the OS, the MFS and the DFS were 0.500 (95\% CI, 0.321-0.778), 0.503 (95\% CI, $0.323-0.784$ ) and 0.521 (95\% CI, 0.338-0.803), respectively. The adjusted survival curves depicted through COX model were shown in Figure 2.

The correlation analysis (Figure 3) showed that serum apoAI level had weak negative correlations with stage (Rho $=-0.115, \mathrm{P}=0.023)$, tumor diameter $(\mathrm{Rho}=-0.137$, $\mathrm{P}=0.013$ ) and lymph node metastasis ( $\mathrm{Rho}=-0.116, \mathrm{P}=0.034$ ). And there was a weak positive correlation between serum level of apoAI and hemoglobin ( $\mathrm{Rho}=0.2, \mathrm{P}=0.001$ ). 
Table 2 The results of multivariate survival analysis

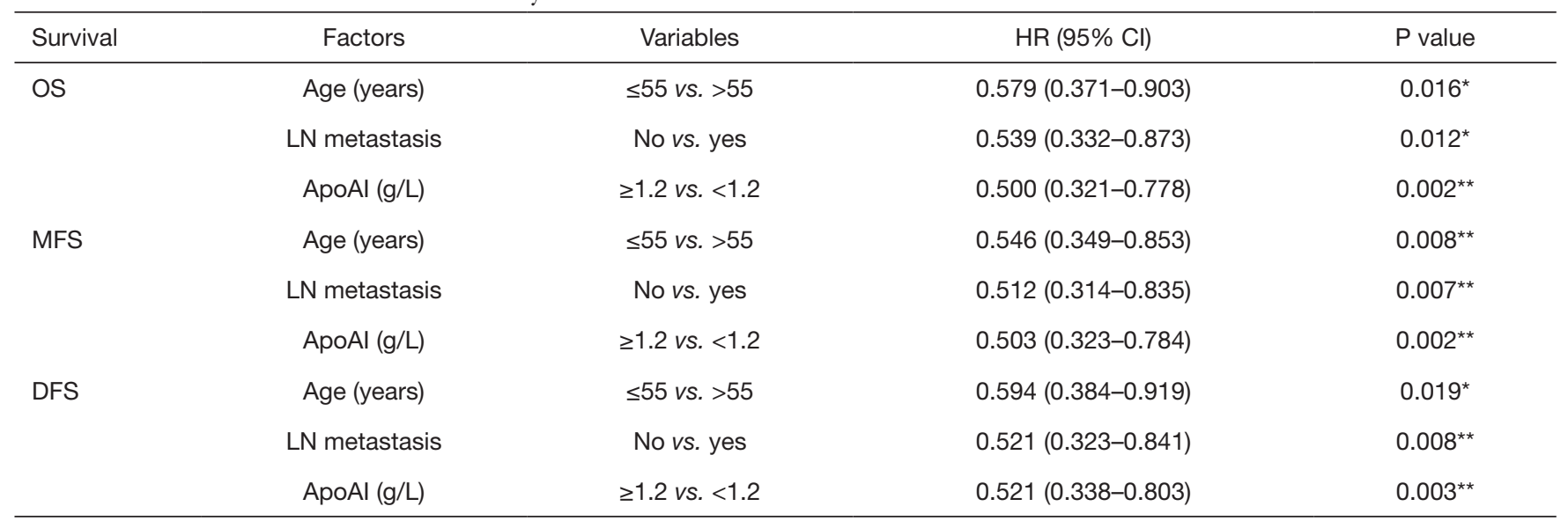

*, $\mathrm{P}<0.05$; ${ }^{*}, \mathrm{P}<0.01$. OS, overall survival; MFS, distant-metastasis-free survival; DFS, disease-free survival; HR, hazard ratio; Cl, confidence interval; LN, lymph node; apoAI, apolipoprotein A-I.
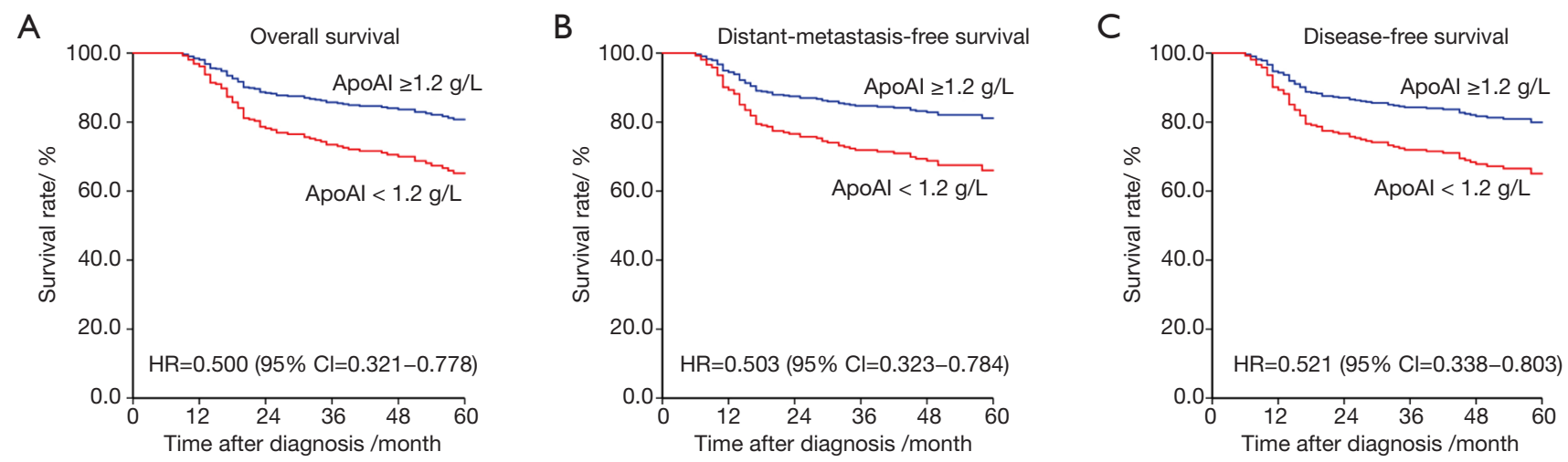

Figure 2 The adjusted survival curves of patients grouped with serum apolipoprotein A-I (apoAI). (A) overall survival; (B) distant-metastasisfree survival; (C) disease-free survival. The covariates included in COX model were age, lymph node metastasis and serum apoAI. HR, hazard ratio; CI, confidence interval.

A

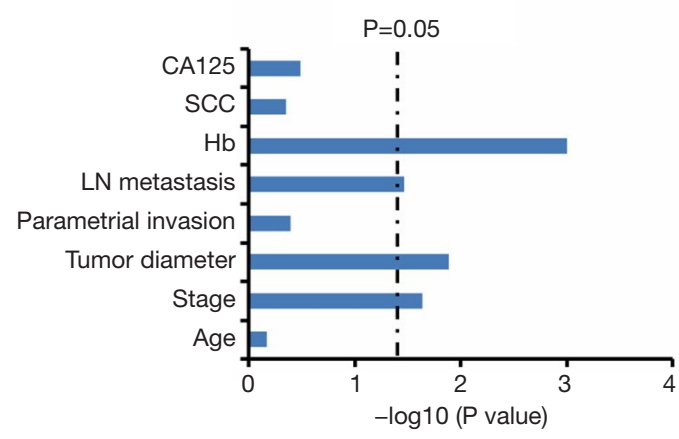

B

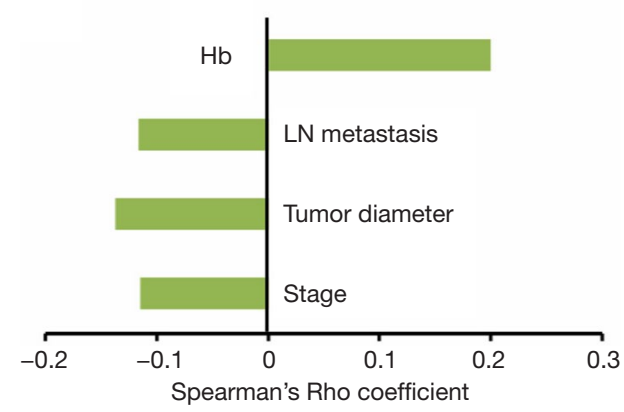

Figure 3 The correlation between serum apolipoprotein A-I (apoAI) and other clinical factors. (A) Statistical significance for each factor; (B) Spearman's Rho coefficient for factors with statistical significance. LN, lymph node; Hb, hemoglobin; SCC, squamous cell carcinoma antigen; CA125, carbohydrate antigen 125 . 


\section{Discussion}

The role of serum lipid components in cancer progression and treatment is one of the research hotspots these days. Until now, there has been only one study focusing on the prognostic value of serum lipid profile in cervical cancer. That study reviewed a small cohort of patients to report that apolipoprotein C-II might be associated with their longterm outcome after CCRT (13). In this study, pretreatment levels of serum lipids were analyzed for their association with prognosis of stages IIB-IVA CSCC, together with known prognosticators of cervical cancer. We found that low level of serum apoAI level predicted a poorer OS, DFS and MFS independently. The results were based on a relatively large-scale cohort and thus reliable. Considering that serum apoAI could be easily detected in daily clinical work, our study provided a practical predictor for locally advanced CSCC treated with CCRT. Except apoAI, none of the serum lipid indexes emerged as predictors of CSCC prognosis.

Besides apoAI, age and lymph node metastasis at initial diagnosis also exhibited predictive independence in this study. These 2 factors have already been proved as prognosticators of cervical cancer in previous studies $(8,14)$. The HRs of age ( $\leq 55 v s$. $>55$ years old) for the OS, the DFS and the MFS were 0.579 (95\% CI, 0.371-0.903), 0.594 (95\% CI, 0.384-0.919) and 0.546 (95\% CI, 0.349-0.853), respectively. The figures of lymph node metastasis (no vs. yes) were 0.539 (95\% CI, 0.332-0.873), 0.521 (95\% CI, $0.323-0.841)$ and 0.512 (95\% CI, 0.314-0.835), respectively. Nevertheless, FIGO stage, tumor diameter, parametrial invasion and serum hemoglobin level failed to exhibit predictive ability on the survivals of the patients. In the correlation analysis, apoAI was showed to have weak correlations with stage, tumor diameter and hemoglobin. It was inferred that these 3 factors might somewhat be influenced by apoAI in these patients. And in addition, the negative correlation between apoAI and stage also supported that low apoAI might be a predictor of CSCC progression.

Due to advance of radiation technique, local control of CSCC, even locally advanced disease, has been greatly improved. According to our results, the LR rate was only $5.4 \%$ after nearly a follow-up time of more than 4 years. In contrast, the DM rate was as high as $25.1 \%$. And all the 81 cancer-deaths harbored DM. It was similar to prior studies that DM had become the primary cause of CSCC-related death. Predictive ability of serum apoAI on the MFS made it an index to evaluate not only patients' survival but also
DM risk. Those with pretreatment low level of serum apoAI might be at a high risk of DM. These patients might achieve survival benefit from intensified systemic therapies aiming to eradicate DM, such as ACT. In other words, serum apoAI could be used to direct individualized treatment of locally advanced CSCC. Actually, before our study, the association between serum apoAI level and DM has been showed in other malignant tumors. For example, Ma et al. found in 443 patients with hepatocellular carcinoma that low serum apoAI was found to be associated with a higher level of circulating tumor cells (15). And in a study by Quan et al., a relationship was seen between low serum apoAI and DM progression of colorectal cancer (16).

The biological fundamentals to explain the impact of serum apoAI on DM are awaited to be elucidated. Yet, there have been studies exhibiting anti-tumor activities of apoAI. Wang et al found that ApoAI concentration was significantly lower in serum samples of patients with esophageal SCC than in those of healthy controls (17). Elevation of serum apoAI level were also reported in ovarian cancer $(18,19)$. In lab studies, Zamanian-Daryoush et al. demonstrated that apoAI could inhibit tumor growth through knocking out apoAI gene of nude mice. Not only that, provision of exogenous apoAI could even lead to shrinkage of xenograft tumor $(20,21)$. There were also studies to explore how apoAI exerted its anti-tumor functions. The possible mechanisms included decreasing tissue concentration of survivin protein, recruiting myeloid-derived suppressor cells, promoting accumulation of macrophages with an M1like anti-tumor phenotype as well as $\mathrm{CD}^{+} \mathrm{T}$ cells, reducing level and activity of metalloproteinase-9, inhibiting tumor angiogenesis, and depressing inflammation (20-24).

The anti-tumor activities of apoAI provided a new idea to manage CSCC. That was to use apoAI as a therapeutic target. Currently, the interfering modalities of serum apoAI are well developed. The lipid-modulating agents, such as niacin and statin, have been maturely used to elevate serum apoAI concentration $(25,26)$. Moreover, a reconstituted human apoAI, the CSL112, was confirmed by Phase 2 clinical trials to be safe when used intravenously $(27,28)$. Hence, it is feasible to treat malignancies through apoAI. Recently, Park et al. have investigated 114,116 healthy individuals and found that niacin intake could reduce the risk of skin SCC (29). And statin was found in a series of studies to improve the prognosis of colon and gynecologic cancers, including cervical cancer (30-32). In this study, patients with locally advanced CSCC and low serum apoAI were more likely to develop DM. ApoAI-elevating therapies 
might be beneficial for these patients. Namely, our results were informative for clinicians to conduct trials focusing on apoAI-targeting treatment in locally advanced CSCC.

Indeed, there were several shortcomings of this study. First, this study was a single-institutional, retrospective study. Second, BT in this study was performed in a 2-dimensional style. It was mainly limited by the technique conditions of our hospital at that time. Hence, we proposed that the results of this study should be popularized with cautions before validation in prospectively enrolled, multi-institutional cohorts treated with BT based on 3-dimensional technique.

In conclusion, low pretreatment level of serum apoAI emerged as an independent predictor of $\mathrm{DM}$ and poor prognosis in patients with stages IIB-IVA CSCC. This finding might help clinicians to direct therapy for eradicating DM and conduct clinical trials on apoAItargeting anti-tumor therapies.

\section{Acknowledgments}

We are grateful to Dr. Christopher Lavender (the Office of International Cooperation and Public Relations, Sun Yat-sen University Cancer Center, Guangzhou, China) for his help in polishing our manuscript.

Funding: This study was supported by the Sun Yatsen University Clinical Research 5010 Program (Grant Number: 2007041).

\section{Footnote}

Conflicts of Interest: All authors have completed the ICMJE uniform disclosure form (available at http://dx.doi. org/10.21037/tcr.2018.11.10). The authors have no conflicts of interest to declare.

Ethical Statement: The authors are accountable for all aspects of the work in ensuring that questions related to the accuracy or integrity of any part of the work are appropriately investigated and resolved. The study was conducted in accordance with the Declaration of Helsinki (as revised in 2013). This study was approved by the Institutional Review Board of the Sun Yat-sen University Cancer Center (YP2008008). Written informed consents were obtained from all individual participants included in the study.

Open Access Statement: This is an Open Access article distributed in accordance with the Creative Commons
Attribution-NonCommercial-NoDerivs 4.0 International License (CC BY-NC-ND 4.0), which permits the noncommercial replication and distribution of the article with the strict proviso that no changes or edits are made and the original work is properly cited (including links to both the formal publication through the relevant DOI and the license). See: https://creativecommons.org/licenses/by-nc-nd/4.0/.

\section{References}

1. Chen W, Sun K, Zheng R, et al. Cancer incidence and mortality in China, 2014. Chin J Cancer Res 2018;30:1-12.

2. Matsuo K, Mandelbaum RS, Machida H, et al. Association of tumor differentiation grade and survival of women with squamous cell carcinoma of the uterine cervix. J Gynecol Oncol 2018;29:e91.

3. Zhou J, Zhang WW, Wu SG, et al. The impact of examined lymph node count on survival in squamous cell carcinoma and adenocarcinoma of the uterine cervix. Cancer Manag Res 2017;9:315-22.

4. Sugiyama T, Fujiwara K, Ohashi Y, et al. Phase III placebo-controlled double-blind randomized trial of radiotherapy for stage IIB-IVA cervical cancer with or without immunomodulator Z-100: a JGOG study. Ann Oncol 2014;25:1011-7.

5. Lee SW, Lee SH, Kim J, et al. Magnetic resonance imaging during definitive chemoradiotherapy can predict tumor recurrence and patient survival in locally advanced cervical cancer: A multi-institutional retrospective analysis of KROG 16-01. Gynecol Oncol 2017;147:334-9.

6. Tangjitgamol S, Katanyoo K, Laopaiboon M, et al. Adjuvant chemotherapy after concurrent chemoradiation for locally advanced cervical cancer. Cochrane Database Syst Rev 2014;(12):CD010401.

7. Pecorelli S. Revised FIGO staging for carcinoma of the vulva, cervix, and endometrium. FIGO Committee on Gynecologic Oncology. Int J Gynaecol Obstet 2009;105:103-4.

8. Rose PG, Java J, Whitney CW, et al. Nomograms Predicting Progression-Free Survival, Overall Survival, and Pelvic Recurrence in Locally Advanced Cervical Cancer Developed From an Analysis of Identifiable Prognostic Factors in Patients From NRG Oncology/ Gynecologic Oncology Group Randomized Trials of Chemoradiotherapy. J Clin Oncol 2015;33:2136-42.

9. Jiang R, Yang ZH, Luo DH, et al. Elevated apolipoprotein A-I levels are associated with favorable prognosis in metastatic nasopharyngeal carcinoma. Med Oncol 2014;31:80. 
10. Zhou T, Zhan J, Fang W, et al. Serum low-density lipoprotein and low-density lipoprotein expression level at diagnosis are favorable prognostic factors in patients with small-cell lung cancer (SCLC). BMC Cancer 2017;17:269.

11. Li X, Tang H, Wang J, et al. The effect of preoperative serum triglycerides and high-density lipoprotein-cholesterol levels on the prognosis of breast cancer. Breast 2017;32:1-6.

12. Zhang $\mathrm{X}$, Zhao $\mathrm{XW}$, Liu DB, et al. Lipid levels in serum and cancerous tissues of colorectal cancer patients. World J Gastroenterol 2014;20:8646-52.

13. Harima Y, Ikeda K, Utsunomiya K, et al. Apolipoprotein $\mathrm{C}-\mathrm{II}$ is a potential serum biomarker as a prognostic factor of locally advanced cervical cancer after chemoradiation therapy. Int J Radiat Oncol Biol Phys 2013;87:1155-61.

14. Tseng JY, Yen MS, Twu NF, et al. Prognostic nomogram for overall survival in stage IIB-IVA cervical cancer patients treated with concurrent chemoradiotherapy. Am J Obstet Gynecol 2010;202:174.e1-7.

15. Ma XL, Gao XH, Gong ZJ, et al. Apolipoprotein A1: a novel serum biomarker for predicting the prognosis of hepatocellular carcinoma after curative resection. Oncotarget 2016;7:70654-68.

16. Quan Q, Huang Y, Chen Q, et al. Impact of Serum Apolipoprotein A-I on Prognosis and Bevacizumab Efficacy in Patients with Metastatic Colorectal Cancer: a Propensity Score-Matched Analysis. Transl Oncol 2017;10:288-94.

17. Wang XP, Li XH, Zhang L, et al. High level of serum apolipoprotein A-I is a favorable prognostic factor for overall survival in esophageal squamous cell carcinoma. BMC Cancer 2016;16:516.

18. Zhang Z, Bast RC Jr, Yu Y, et al. Three biomarkers identified from serum proteomic analysis for the detection of early stage ovarian cancer. Cancer Res 2004;64:5882-90.

19. Sun Y, Meng H, Jin Y, et al. Serum lipid profile in gynecologic tumors: a retrospective clinical study of 1,550 patients. Eur J Gynaecol Oncol 2016;37:348-52.

20. Zamanian-Daryoush M, Lindner D, Tallant TC, et al. The cardioprotective protein apolipoprotein A1 promotes potent anti-tumorigenic effects. J Biol Chem 2013;288:21237-52.

21. Su F, Kozak KR, Imaizumi S, et al. Apolipoprotein A-I (apoA-I) and apoA-I mimetic peptides inhibit tumor development in a mouse model of ovarian cancer. Proc Natl Acad Sci U S A 2010;107:19997-20002.

22. Sag D, Cekic C, Wu R, et al. The cholesterol transporter ABCG1 links cholesterol homeostasis and tumour immunity. Nat Commun 2015;6:6354.
23. Yi ZF, Cho SG, Zhao H, et al. A novel peptide from human apolipoprotein(a) inhibits angiogenesis and tumor growth by targeting c-Src phosphorylation in VEGFinduced human umbilical endothelial cells. Int J Cancer 2009; 124:843-52.

24. Ren K, Lu YJ, Mo ZC, et al. ApoA-I/SR-BI modulates S1P/S1PR2-mediated inflammation through the PI3K/ Akt signaling pathway in HUVECs. J Physiol Biochem 2017;73:287-96.

25. Pang J, Chan DC, Hamilton SJ, et al. Effect of niacin on high-density lipoprotein apolipoprotein A-I kinetics in statin-treated patients with type 2 diabetes mellitus. Arterioscler Thromb Vasc Biol 2014;34:427-32.

26. Kakuda H, Matoba M, Nakatoh H, et al. Effects of change in high-density lipoprotein cholesterol by statin switching on glucose metabolism and renal function in hypercholesterolemia. J Clin Lipidol 2015;9:709-15.

27. Gibson CM, Korjian S, Tricoci P, et al. Rationale and design of Apo-I Event Reduction in Ischemic Syndromes I (AEGIS-I): A phase 2b, randomized, placebo-controlled, dose-ranging trial to investigate the safety and tolerability of CSL112, a reconstituted, infusible, human apoA-I, after acute myocardial infarction. Am Heart J 2016;180:22-8.

28. Tricoci P, D'Andrea DM, Gurbel PA, et al. Infusion of Reconstituted High-Density Lipoprotein, CSL112, in Patients With Atherosclerosis: Safety and Pharmacokinetic Results From a Phase 2a Randomized Clinical Trial. J Am Heart Assoc 2015;4:e002171.

29. Park SM, Li T, Wu S, et al. Niacin intake and risk of skin cancer in US women and men. Int J Cancer 2017;140:2023-31.

30. Voorneveld PW, Reimers MS, Bastiaannet E, et al. Statin Use After Diagnosis of Colon Cancer and Patient Survival. Gastroenterology 2017;153:470-9.e4.

31. Vogel TJ, Goodman MT, Li AJ, et al. Statin treatment is associated with survival in a nationally representative population of elderly women with epithelial ovarian cancer. Gynecol Oncol. Gynecol Oncol 2017;146:340-5.

32. Song MK, Shin BS, Ha CS, et al. Would Lipophilic Statin Therapy as a Prognostic Factor Improve Survival in Patients With Uterine Cervical Cancer? Int J Gynecol Cancer 2017;27:1431-7.

Cite this article as: Chang $\mathrm{H}$, Chen $\mathrm{K}$, Lin $\mathrm{YZ}$, Tao $\mathrm{YL}$, Liu Y, Cao XP, Ye WJ. Prognostic value of serum apolipoprotein A-I in locally advanced cervical squamous cell carcinoma treated with radical chemoradiotherapy. Transl Cancer Res 2018;7(6):1474-1481. doi: 10.21037/tcr.2018.11.10 